\title{
Hydrogeological characterization throughout deep geophysical investigations in the Verrès plain (Aosta Valley, north-western Italian Alps)
}

\section{Caratterizzazione idrogeologica mediante indagini geofisiche profonde sulla piana di Verrès (Valle d'Aosta)}

Pietro Capodaglio, Mario Naldi, Fulvio Simonetto

Riassunto: A fronte di una disponibilità straordinaria di acqua dolce, garantita dalla copertura glaciale e dalla fusione stagionale delle nevi in quota, le condizioni di ricerca idrogeologica in Valle d'Aosta non sono ovunque favorevoli.

La maggior parte del territorio è spiccatamente montuoso con prevalenza di litotipi metamorfici e subordinati depositi glaciali. Corpi idrici sotterranei di una certa rilevanza risultano localizzati quasi esclusivamente sul fondovalle principale, ove hanno avuto massima intensità i fenomeni - prima di escavazione glaciale e successivamente di sedimentazione in ambito glaciale, lacustre e fluviale - che hanno consentito la deposizione di spessori significativi di materiali permeabili per porosità. Nonostante l'importanza di tali corpi idrici, nelle zone di fondovalle il sottosuolo risulta tuttora in parte sconosciuto.

ARPA Valle d'Aosta monitora i principali acquiferi ai sensi della vigente normativa (D.Lgs.30/09).

Nella presente nota viene esaminato l'acquifero della piana di Verrès, nella bassa Valle d'Aosta, mai indagato precedentemente dal punto di vista geofisico. Scopo principale dell'indagine

Parole chiave: fondovalle alluvionale, monitoraggio acque sotterranee, indagini geofisiche.

Keywords: alluvial bottom valley, groundwater monitoring, geophysical investigations.

Pietro CAPODAGLIO 曩”

Agenzia Regionale per la Protezione dell'Ambiente della Valle d'Aosta Sezione ASSC

Loc .Grande Charrière 44, 11020 St. Christophe (AO)

p.capodaglio@arpa.vda.it

\section{Fulvio SIMONETTO}

Agenzia Regionale per la Protezione dell'Ambiente della Valle d'Aosta Sezione ASSC

11020 St. Christophe (AO)

\section{Mario NALDI}

Techgea srl

Loc. Amérique 9, 11020 Quart (AO)

Ricevuto/Received: 07 February 2017-Accettato/Accepted: 20 March 2017 Pubblicato online/Published online: 31 March 2017

This is an open access article under the CC BY-NC-ND license: http://creativecommons.org/licenses/by-nc-nd/4.0/

(C) Associazione Acque Sotterranee 2017 era valutare, in via preliminare, le possibilità di ricerca della risorsa idrica sotterranea in eventuali acquiferi profondi al momento non noti.

Con l'utilizzo combinato di diversi metodi geofisici (ERT, TDEM, HVSR e Re.Mi.) si è ricostruita la geometria del sistema acquifero profondo individuando, con la migliore approssimazione possibile, la profondità del basamento lapideo.

Abstract: Although fresh water availability in the Aosta Valley (north-western Italian Alps) is generally granted by glaciers and snow seasonal melting at high altitudes, bydrogeological conditions are not favorable everywhere.

Most part of the territory is typically mountainous, with prevailing metamorphic rocks and, secondarily, glacial deposits.

Relevant ground water bodies can be found only in the main bottom valley, where glacial excavation, fluvio-glacial and lacustrine sedimentation had maximum intensity, allowing the deposition of important thickness of porous materials. Nevertheless, the geological knowledge of the subsurface is here still poor.

These groundwater bodies are monitored by the Environmental Protection Agency of the Aosta Valley Region (ARPA Valle d'Aosta), according to the Italian law (D.Lgs.30/09).

This study deals with geophysical investigations in the Verrès plain aquifer (southern Aosta Valley). The main goal of the study has been the first evaluation of groundwater research in potential deep aquifers. Different geophysical methodologies were applied (ERT, TDEM, HVSR, and Re.Mi.), in order to identify the deep aquifer geometry and the rock basement depth.

\section{Introduzione}

Lo studio si propone di sopperire, almeno in parte, ad alcune lacune conoscitive riguardanti la parte più profonda dei depositi che colmano un settore del fondovalle valdostano, carente di dati geognostici pregressi e mai precedentemente indagato dal punto di vista geofisico. Più in dettaglio, si è voluto valutare e caratterizzare i seguenti aspetti:

- La profondità e l'andamento di massima del substrato roccioso (mai raggiunto direttamente in perforazione da nessun pozzo o sondaggio geognostico);

- La tipologia e gli spessori dei depositi presenti al di sotto dei $100 \mathrm{~m}$ dal piano campagna, tra il substrato e la parte più superficiale dei sedimenti alluvionali;

- La potenziale presenza di acquiferi profondi. 
Lutilizzo di metodi geofisici per la valutazione degli spessori delle coltri detritiche di elevato spessore (centinaia di metri) costituisce un approccio particolarmente efficace all'individuazione di potenziali acquiferi profondi. Lunico esempio noto in Val d'Aosta di indagini geofisiche profonde mirate alla valutazione degli spessori della coltre detritica di fondovalle per caratterizzazione idrogeologica, si riferisce allo studio di Armando E. e Dal Piaz G.V. (1970). Gli Autori hanno analizzato l'assetto litostratigrafico della piana di Aosta utilizzato il metodo sismico a rifrazione con stendimenti di lunghezza compresa tra 500 e 1000 e utilizzo dell'esplosivo. L'interpretazione dei dati (datata ai primi anni '70, e che quindi si è avvalsa in via del metodo delle dromocrone) ha consentito di localizzare il substrato lapideo ad oltre 300 $\mathrm{m}$ di profondità, con buone prospettive di localizzazione di acquiferi profondi. Nel 2011, nella stessa zona di indagine, Techgea Srl ha condotto una indagine geoelettrica profonda in adiacenza alla pista aeroportuale dello scalo Corrado Gex di Aosta (Techgea Srl 2011). L'indagine geoelettrica ha previsto la realizzazione di uno stendimento lineare di circa $940 \mathrm{~m}$ di lunghezza, con il raggiungimento di una profondità di circa $200 \mathrm{~m}$. I risultati dell'indagine non hanno consentito di localizzare il substrato lapideo, e ciò conferma i risultati dell'indagine sismica prima citata (Armando e Dal Piaz 1970), ma aggiunge un particolare di notevole rilevanza ai fini idrogeologici. Si è individuata, infatti, una struttura sedimentaria a bassa resistività elettrica, congruente con la presenza di depositi fini, limosi o limoso-argillosi (depositi di probabile origine lacustre), interposta tra i depositi alluvionali superficiali ed un orizzonte più resistivo profondo (acquifero in pressione).

Per raggiungere profondità congruenti con quelle attese per il substrato lapideo sepolto (almeno 300-400 m) è stato necessario valutare l'utilizzo di altri metodi di indagine, in accordo, tuttavia, con le limitazioni imposte dalle condizioni sito-specifiche. In particolare, l'utilizzo di metodi quali la sismica a riflessione o la tecnica audiomagnetotellurica risultano inapplicabili per l'impossibilità di utilizzare sorgenti di energia efficienti (esplosivo) e per l'elevato disturbo elettromagnetico di origine antropica, che si sovrappone al segnale elettromagnetico generato dalle correnti magnetotelluriche. Si è quindi scelto di utilizzare dei metodi di indagine a minor grado di risoluzione ma in grado di fornire, secondo sondaggi "puntuali" delle indicazioni semi-quantitative circa la profondità del substrato. In particolare, si è fatto riferimento al metodo HVSR che, grazie alla registrazione continuativa degli eventi "vibratori" o "sismici" su tempi molto lunghi ("microtremori" registrati continuativamente per un periodo di 4 ore), consente un campionamento di segnali sismici a differente frequenza, da cui ricavare i picchi di segnale relativi alla frequenza di risonanza che identificano i maggiori contrasti di impedenza (segnatamente il passaggio dalla coltre detritica al substrato rigido). Un esempio di indagine simile al caso in esame è riferito da Mele et Al. (2016); gli Autori hanno utilizzato il metodo HVSR per localizzare l'andamento del substrato sismico in
Val Chiavenna (Sondrio), in condizioni ambientali molto simili a quelle della Piana di Verrès e con notevoli profondità del substrato (oltre $200 \mathrm{~m}$ ). Caso analogo viene presentato da Brown et Al (2013) per la localizzazione del substrato lapideo secondo una griglia di punti su maglia regolare in un'area pianeggiante in Connecticut (USA). Oltre al metodo sismico HVSR è stato applicato il metodo elettromagnetico TDEM; molto simile al metodo magnetotellurico, il metodo TDEM registra il tempo di decadimento di un campo elettromagnetico indotto artificialmente da una bobina di forma quadrata all'interno del quale circola corrente. La possibilità di controllare la sorgente di energia riduce, in via teorica, il disturbo indotto da fonti elettromagnetiche esterne. In realtà, i limiti imposti dagli spazi disponibili nell'area di fondovalle (fondovalle (la bobina ha dimensioni minime di $40 \times 40 \mathrm{~m}$ fino a 200x200 m) in combinazione con i forti campi elettromagnetici creati dalle linee aeree ad alta tensione o da macchinari a motore elettrico, rende talora inutilizzabili i risultati, salvo alcune eccezioni. La combinazione incrociata di tutti i metodi di indagine utilizzati, opportunamente calibrata con le conoscenze geologiche e stratigrafiche del sito, ha infine permesso una ricostruzione dell'apparato sedimentario della Piana di Verrès, evidenziando nuove prospettive di sviluppo delle strutture acquifere profonde.

\section{Area di studio}

La Piana di Verrès è situata nella bassa Valle d'Aosta e si estende longitudinalmente per circa $13 \mathrm{~km}$ in direzione Nord-Ovest - Sud-Est. Ai sensi del D.Lgs.30/09 l'acquifero in essa ospitato è classificabile nella tipologia "Alluvioni vallive" (Fig. 1) e, più in particolare, negli "Acquiferi a flusso intergranulare significativo superficiale".

Dal punto di vista morfologico la piana è delimitata dalla stretta di Montjovet a monte (ad un'altitudine di circa $400 \mathrm{~m}$ s.l.m.) e dalla stretta di Bard a valle (ad un'altitudine di circa $350 \mathrm{~m}$ s.l.m., per un'estensione areale complessiva di circa $10 \mathrm{~km}^{2}$. In senso trasversale, essa è chiaramente confinata da imponenti pareti rocciose, sovente a giacitura sub-verticale, culminanti in cime superiori ai $2500 \mathrm{~m}$ s.l.m., raggiungendo un'ampiezza massima di circa $1200 \mathrm{~m}$ nella sua porzione centrale, in corrispondenza della cittadina di Verrès. La piana (zona centrale gialla e verde in Fig. 2), è caratterizzata su ambo i versanti da ampi conoidi, in corrispondenza dei quali risultano situati i principali centri abitati.

La piana ricade nel bacino idrografico montano della Dora Baltea ed è centrata sul punto di confluenza in quest'ultima del Torrente Evançon, suo principale affluente nell'area avente origine dal massiccio del Monte Rosa; inoltre, la Dora Baltea accoglie le acque di un cospicuo numero di affluenti secondari a regime glaciale o nivo-glaciale, sia in sinistra che in destra orografica (Fig. 3).

Dal punto di vista geologico la piana di Verrès si sviluppa nell'ambito dei domini Pennidico e Austroalpino, caratterizzati da strutture fortemente piegate con marcato metamorfismo e associate a grandi sovrascorrimenti; più in particolare, la zona è interessata dal sovrascorrimento della Zona Sesia- 


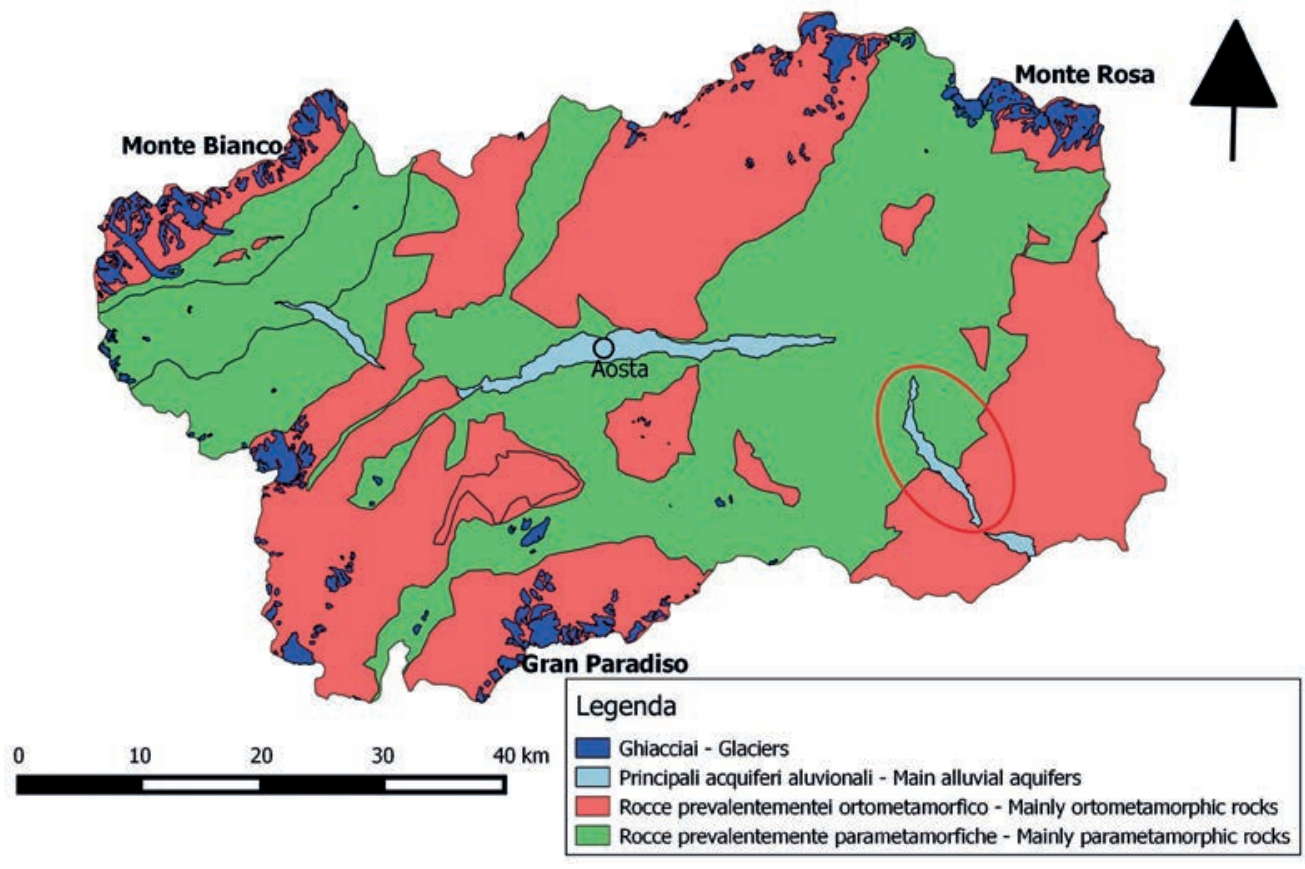

Fig. 1 - Simplified geological map of the Aosta Valley and his aquifers according to the Italian regulation DS.Lgs.30/09. The area of interest is highlighted by the red ellipse.

Fig. 1 - Carta geologica semplificata del territorio valdostano e ubicazione dei principali acquiferi ai sensi del D.Lgs.30/09. L'ellisse rossa individua l'area oggetto del presente lavoro.

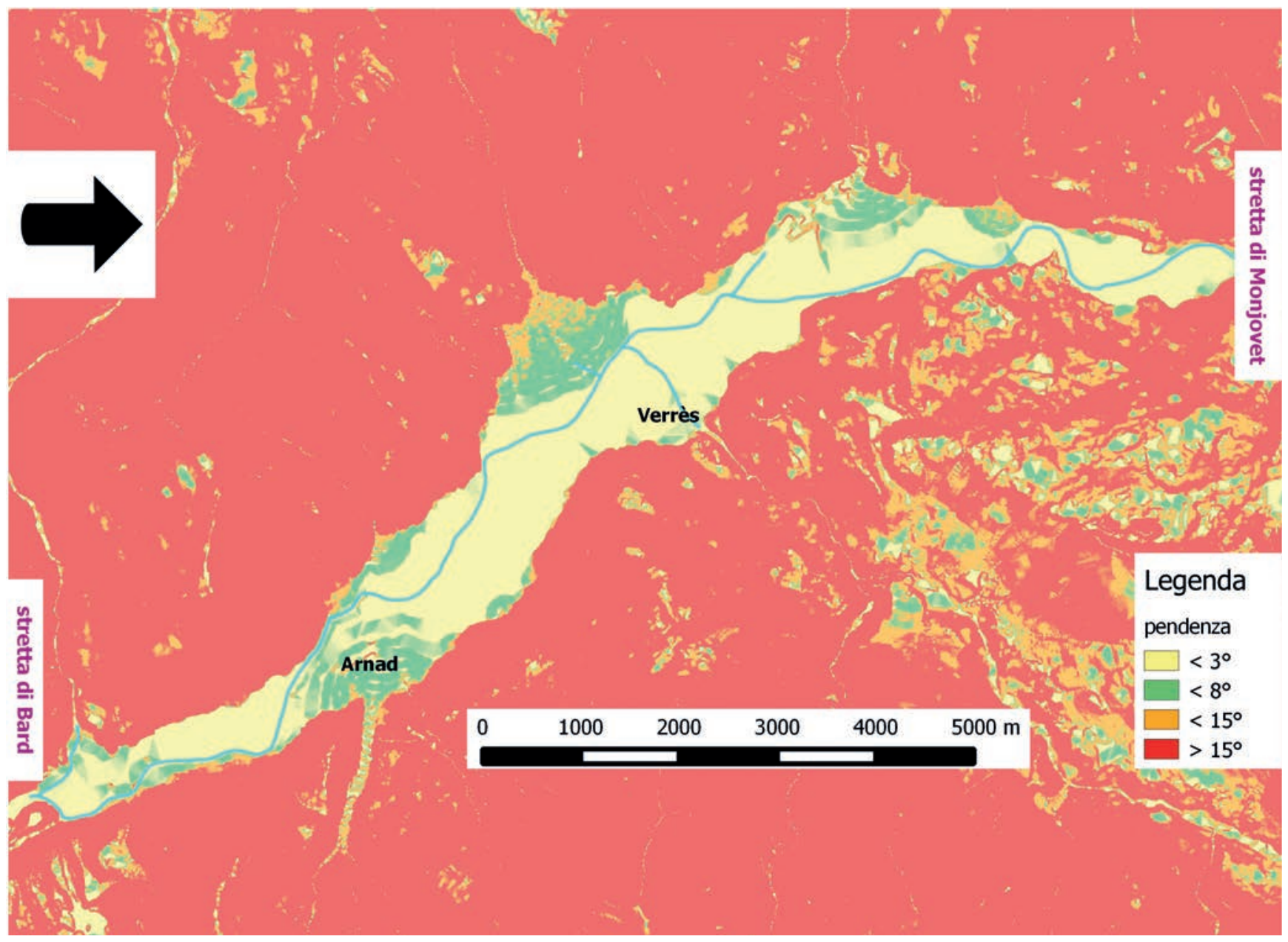

Fig. 2 - Slope map of the Verrès plain.

Fig. 2 - Carta delle pendenze della piana di Verrès. 


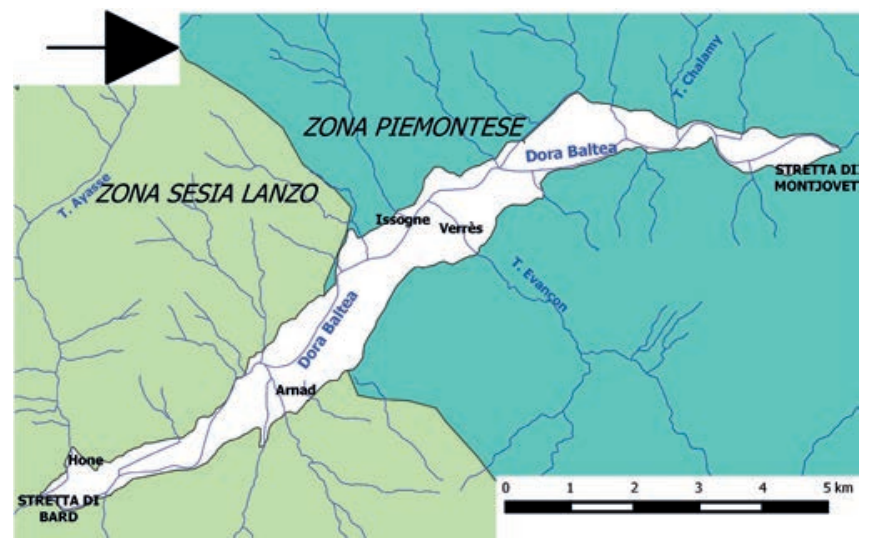

Fig. 3 - Simplified geological map.

Fig. 3 - Carta geologica semplificata.

Lanzo (Austroalpino) - affiorante sui versanti del tratto di valle (sudest) della piana e costituita da micascisti, eclogitici e gneiss minuti - sulla Zona Piemontese (Pennidico) affiorante nel tratto di monte e costituita da calcescisti e serpentiniti. Dal punto di vista idrogeologico, i litotipi afferenti ai due domini presentano caratteristiche del tutto simili tra di loro.

La captazione delle acque sotterranee riguarda per lo più alcuni pozzi concentrati nel piccolo polo industriale di Verrès, ubicato nei pressi della confluenza del T. Evançon nella Dora Baltea, ove l'emungimento è quantificabile in alcuni milioni di metri cubi annui. I prelievi idropotabili da falda risultano di entità molto minore (attorno ai 25 $\div 50.000 \mathrm{~m}^{3} /$ annui), provenienti esclusivamente da un pozzo dell'acquedotto di Verrès, dal momento che la maggior parte degli approvvigionamenti deriva da sorgenti ubicate in quota. Infine, risultano non quantificabili e trascurabili - anche perché a carattere stagionale - i prelievi ad uso irriguo, per il quale sono utilizzati soprattutto corsi d'acqua superficiali presenti sui versanti (localmente detti " $r \hat{u}$ ").

\section{Monitoraggio idrogeologico}

Lacquifero freatico ospitato nella piana di Verrès è monitorato da ARPA VdA dal 2005 secondo le normative vigenti (D.Lgs.152/99 sino al 2009, D.lgs.30/09 successivamente) sia dal punto di vista quantitativo (livello della falda) che qualitativo (prelievi e successive analisi di laboratorio).

Lo stato quantitativo, in mancanza di metodologie specifiche fornite dalla normativa, è valutato confrontando il trend della serie storica disponibile, al fine di individuare eventuali abbassamenti anomali eventualmente ascrivibili a prelievi eccessivi.

La soggiacenza varia tra pochi metri dal piano campagna nelle zone prossime alla Dora Baltea sino a valori superiori ai 20 m. (Fig. 4).

In Fig. 5 è illustrato a titolo esemplificativo un diagramma freatimetrico, derivante da misure piezometriche puntuali a cadenza mensile, relativo al pozzo idropotabile dell'acquedotto di Verrès, ubicato in corrispondenza del conoide del $\mathrm{T}$. Evançon, di cui si ha attualmente a disposizione una serie

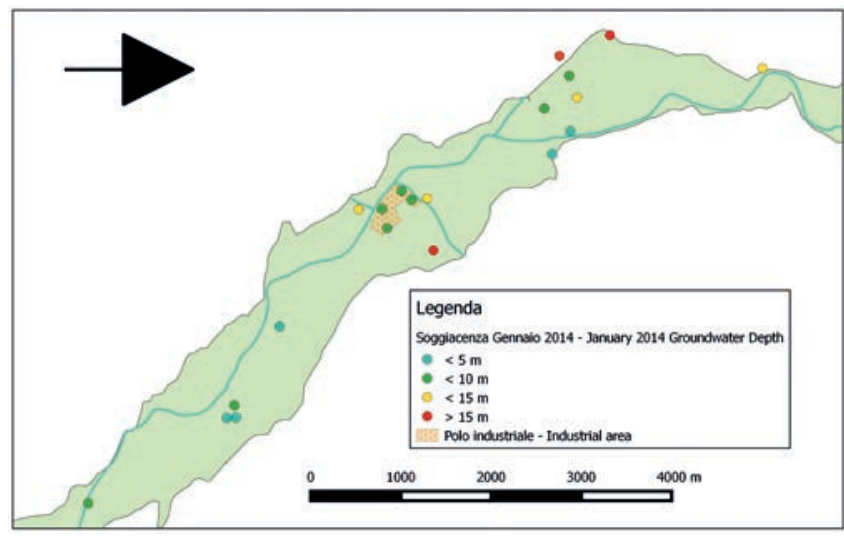

Fig. 4 - Groundwater depth map.

Fig. 4 - Carta della soggiacenza della falda.

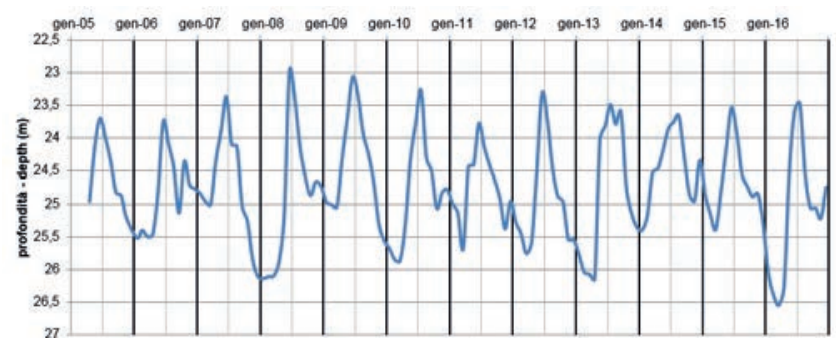

Fig. 5 - Measured groundwater levels in a well in Verrès.

Fig. 5 - Diagramma freatimetrico relativo a un pozzo di Verrès.

storica di 12 anni: si evidenzia un regime stagionale piuttosto regolare (massimo estivo e minimo invernale, conseguente alla fusione nivale tardo primaverile) con un'escursione stagionale dell'ordine dei 2-3 m. I livelli appaiono sostanzialmente stabili su scala pluriennale, ad indicare uno stato quantitativo "buono" ai sensi del suddetto D.Lgs.30/09.

La carta delle isofreatiche (Fig. 6) mostra un andamento di flusso regolare a direzione NW-SE e gradiente idraulico pari a 0,003. Quest'ultimo decresce lievemente in corrispondenza della parte centrale della piana, che qui assume la sua massima larghezza, e dove sono localizzati alcuni pozzi.

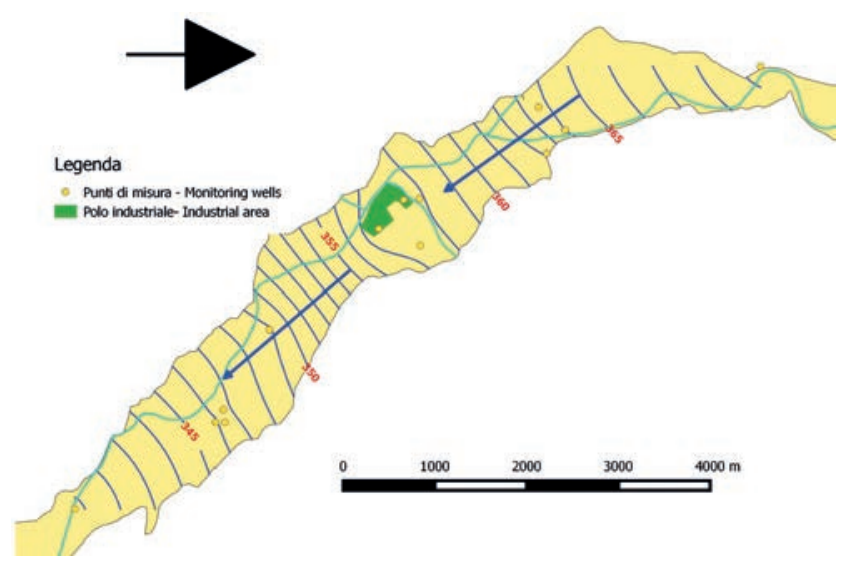

Fig. 6 - Isophreatic map - November 2014.

Fig. 6 - Carta delle isofreatiche - Novembre 2014. 
La falda si trova in rapporto di sostanziale equilibrio con la Dora Baltea, mentre i corsi d'acqua laterali impostati in corrispondenza dei conoidi (es. il T. Evançon) alimentano la falda.

Per quanto riguarda lo stato qualitativo (stato chimico), i prelievi nel corso degli anni non hanno mai evidenziato - per nessun parametro e in nessun punto della rete di monitoraggio, costituita da n. 6 piezometri - superamenti dei limiti normativi, pertanto lo stato del corpo idrico è classificabile senz'altro come "buono"; a riprova si evidenzia che sull'area in oggetto non è mai stato aperto alcun procedimento per sito contaminato ai sensi del D.Lgs.152/06 Parte Quarta Titolo V.

Dal punto di vista idrogeochimico, le acque appartengono alla facies bicarbonato-calcica come si può desumere dal diagramma di Fig. 7, relativo ai punti di prelievo della rete di monitoraggio ARPA nella campagna del Giugno 2014.

In definitiva l'area di studio risulta caratterizzata dal fatto di essere ampiamente sottoutilizzata per prelievi idrici rispetto alle proprie potenzialità e di essere del tutto priva di fenomeni di inquinamento delle acque sotterranee.

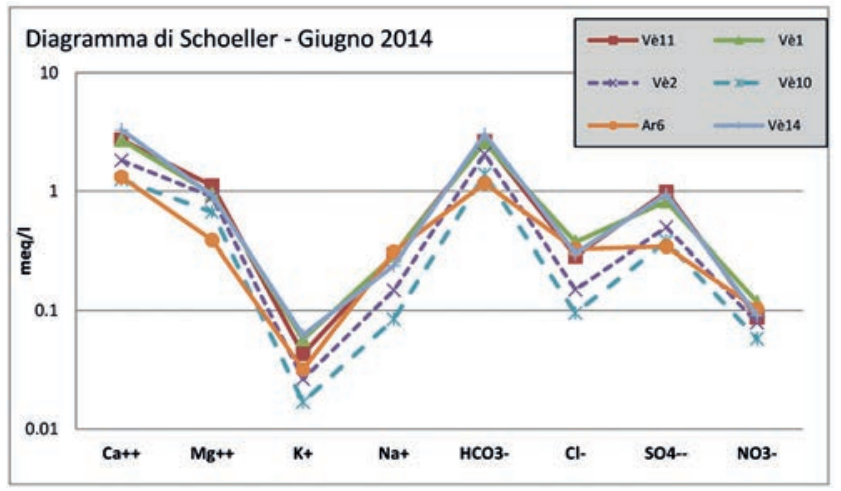

Fig. 7 - Schoeller diagram - June 2014.

Fig. 7 - Diagramma di Schoeller- Giugno 2014.

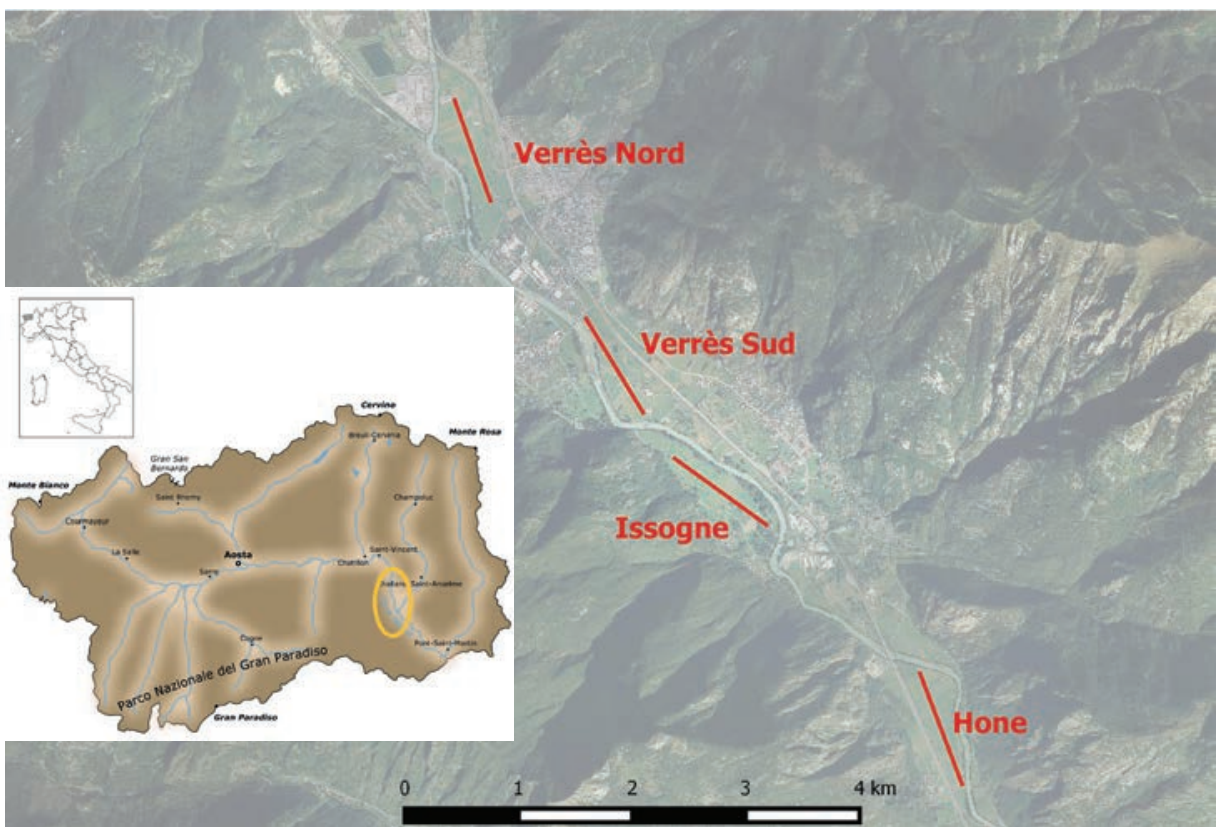

\section{Indagini geofisiche}

Nel 2013 ARPA VdA ha commissionato a Techgea srl l'effettuazione di un totale di n. 4 stendimenti geofisici in zone della piana di Verrès, con metodologie anche innovative per il raggiungimento dell'obiettivo di indagine.

L'indagine aveva i seguenti scopi: a) valutare le caratteristiche della coltre detritica alluvionale della piana; b) individuare un presunto orizzonte profondo a granulometria fine di origine lacustre, la cui esistenza è suggerita dalla storia geologica recente della Valle d'Aosta, che ha visto il succedersi di una successione di depositi fluvio-glaciali, lacustri, alluvionali e di conoide depostisi in epoca quaternaria su un basamento cristallino modellato dall'azione del ghiacciaio Balteo.

Tale livello fine rivestirebbe un'importanza fondamentale ai fini idrogeologici, in quanto potrebbe costituire uno strato di separazione tra l'acquifero alluvionale superficiale, attualmente captato e monitorato, e un presunto acquifero profondo ospitato in depositi morenici posti sotto lo strato lacustre, in appoggio al basamento lapideo.

L'individuazione delle località ove eseguire le indagini (nei comuni di Verrès, Issogne e Hône; Fig. 8) è stata dettata da condizioni logistiche, ovvero dalla possibilità di effettuare stendimenti sufficientemente lunghi (dell'ordine di $1 \mathrm{~km}$ ciascuno) compatibilmente con l'antropizzazione del territorio.

Oltre a quest'ultima condizione ostativa, va premesso che l'area in esame presenta altri ostacoli alla pianificazione di indagini geofisiche profonde, quali: a) un rumore elettromagnetico ad ampio spettro di frequenza generato da svariati fattori (linee elettriche AT, insediamenti industriali e urbani, sottoservizi, motori elettrici, cavi elettrici aerei/interrati, antenne radio, strutture metalliche in movimento, ecc.); b) un generico disturbo sismico generato da traffico veicolare dell'autostrada e della statale, dal rumore prodotto dalla Dora Baltea e affluenti, ecc. A causa di tali fattori, non è stato tra l'altro possibile effettuare stendimenti trasversali alla valle.
Fig. 8 - Location of geophysical investigations (red lines).

Fig. 8 - Ubicazione degli stendimenti geofisici effettuati (linee rosse). 


\section{Metodi utilizzati}

Sono stati utilizzati differenti metodi geofisici, tra loro complementari:

1. metodo geoelettrico multi-elettrodo (Electrical Resistivity Tomography - ERT);

2. sondaggi di «rumore sismico» (Horizontal to Vertical Spectral Ratio - HVSR);

3. sondaggi elettromagnetici (Time Domain Electro Magnetic - TDEM);

4. indagine di "Refraction Microtremor" (Re.Mi.).

In particolare, sui siti di Verrès Sud e Issogne sono stati utilizzati i primi due metodi, sul sito di Verrès Nord i primi tre metodi mentre a Hône tutti i metodi previsti.

Le metodologie ERT e TDEM determinano le caratteristiche di resistività elettrica del sottosuolo, consentendo di valutare sia le variazioni stratigrafiche verticali (attribuibili a passaggi litologici oppure a cambi delle proprietà fisiche dell'ammasso roccioso, quali fratturazione o contenuto d'acqua) sia quelle laterali (attribuibili a variazioni sedimentarie o a discontinuità tettoniche).

Il metodo ERT è una tecnica di indagine geofisica di tipo attivo, con la quale si misura, tra due punti (elettrodi) in superficie, la differenza di potenziale determinata dall'iniezione di corrente continua nel sottosuolo effettuata attraverso l'uso di una seconda coppia di elettrodi. Il principio fisico su cui si basano le misure è la legge di Ohm: viene determinata la resistività elettrica propria del mezzo in funzione dell'intensità del campo elettrico applicato, della densità di corrente e della corrispondente differenza di potenziale. Le caratteristiche di resistività elettrica sono strettamente correlate alle caratteristiche chimico-fisiche del mezzo attraversato e, per tale motivo, la sezione geoelettrica fornisce una visione del sottosuolo diagnostica ed utile. Inoltre tale tecnica - a differenze delle altre due - offre il vantaggio (non secondario nell'area in esame) di non risentire di eventuali fonti di disturbo elettromagnetico. D'altro canto, l'ERT non consente normalmente il raggiungimento di grandi profondità, essendo queste ultime funzione della lunghezza degli stendimenti (nella presente indagine essa ha consentito una caratterizzazione sino a ca. $200 \mathrm{~m}$ ); tuttavia questa tecnica può essere utilizzata per tarare i risultati derivanti dagli altri due metodi.

I sondaggi elettromagnetici TDEM sono anch'essi una tecnica attiva (in quanto si fa uso d un trasmettitore) nel dominio di tempo, basata sulla misura del decadimento di voltaggio, successivamente convertito in resistività apparente, generato da correnti elettriche vacanti (campo elettromagnetico secondario) prodotte a loro volta dalla circolazione in profondità di deboli correnti elettriche indotte artificialmente (campo elettromagnetico primario). La propagazione del campo elettromagnetico primario viene ottenuta per induzione in seguito alla circolazione di corrente alternata in una bobina, costituita da un cavo elettrico, disposta in forma circolare o a quadrato sul terreno. Dall'allineamento di più sondaggi puntuali interdistanti 50-
$100 \mathrm{~m}$ si ottiene una sezione del sottosuolo simile a quanto prodotto dalla elaborazione ERT di cui sopra (anche se non come modello 2D). La profondità di indagine (dipendente dalla configurazione strumentale prescelta, dalle dimensioni della bobina trasmittente, dalle caratteristiche di resistività elettrica del sottosuolo, dai tempi di acquisizione e dal rumore elettromagnetico di fondo) raggiunge in condizioni ottimali (ovvero in assenza di disturbi elettromagnetici) profondità di alcune centinaia di metri.

La tecnica di indagine sismica passiva HVSR si basa sulla determinazione, mediante sismometro, della frequenza fondamentale di vibrazione (o frequenza di risonanza) del sedimento di copertura a partire da registrazioni di microtremore sismico (principalmente costituito da onde sismiche superficiali). Una volta nota la frequenza di picco, per ricavare con una semplice relazione la profondità del substrato, è necessario conoscere la velocità media delle onde di taglio del profilo di sottosuolo attraversato. A tal scopo, per calibrare il metodo HVSR, è stata eseguita una prova Re.Mi. (Refraction Microtremor) sul sito di Hône, in virtù dell'ampio spazio ivi disponibile, su dispositivo circolare ad ampio raggio per raggiungere la massima profondità possibile (circa $150 \mathrm{~m}$ ). Il metodo di indagine sismico ReMi (Refraction Microtremor) è caratterizzato, a differenza del metodo MASW, da un tipo di acquisizione "passivo" delle onde superficiali che vengono generate dal rumore di fondo (detto anche "microtremore") prodotto da sorgenti naturali ed antropiche (traffico, attività industriali, acque correnti, vento, etc.) e che vengono registrate $\mathrm{da}$ uno stendimento (in questo caso circolare) composto da geofoni verticali con frequenza di $4.5 \mathrm{~Hz}$ posti a distanze regolari l'uno dall'altro (distanza intergeofonica). La tecnica ReMi, indagando bande spettrali con basse frequenze, permette di avere informazioni sull'andamento delle Vs al di sotto del piano campagna del sito, ed in particolar modo di maggior dettaglio della porzione di sismo-strati più profonda del sottosuolo. I risultati di tale prova sono stati utilizzati per l'interpretazione di tutte le indagini HVSR.

\section{Risultati \\ Sito di Verrès Nord}

Sono stati eseguite le seguenti indagini (Fig. 9):

- n. 1 sondaggio elettromagnetico TDEM con loop 40x40 $\mathrm{m}$, intensità massima $20 \mathrm{~A}$ frequenza di ripetizione da $500 \mathrm{~Hz}$ a $0.5 \mathrm{~Hz}$;

- $\quad$ n. 2 prove sismiche HVSR con tempo di registrazione di 4 ore;

- $\quad$ n. 1 stendimento geoelettrico lungo 950 m (96 elettrodi spaziati di $10 \mathrm{~m}$ ) in configurazione mista Wenner Schlumberger.

Nella porzione di valle dello stendimento si rilevano, al di sotto dei depositi alluvionali recenti, materiali ad alta resistività, interpretabili come depositi coalescenti di conoide ad elevata energia (blocchi lapidei), coalescenti del torrente Evançon, che rappresentano contributi laterali ai depositi fluvio-glaciali di fondovalle. 

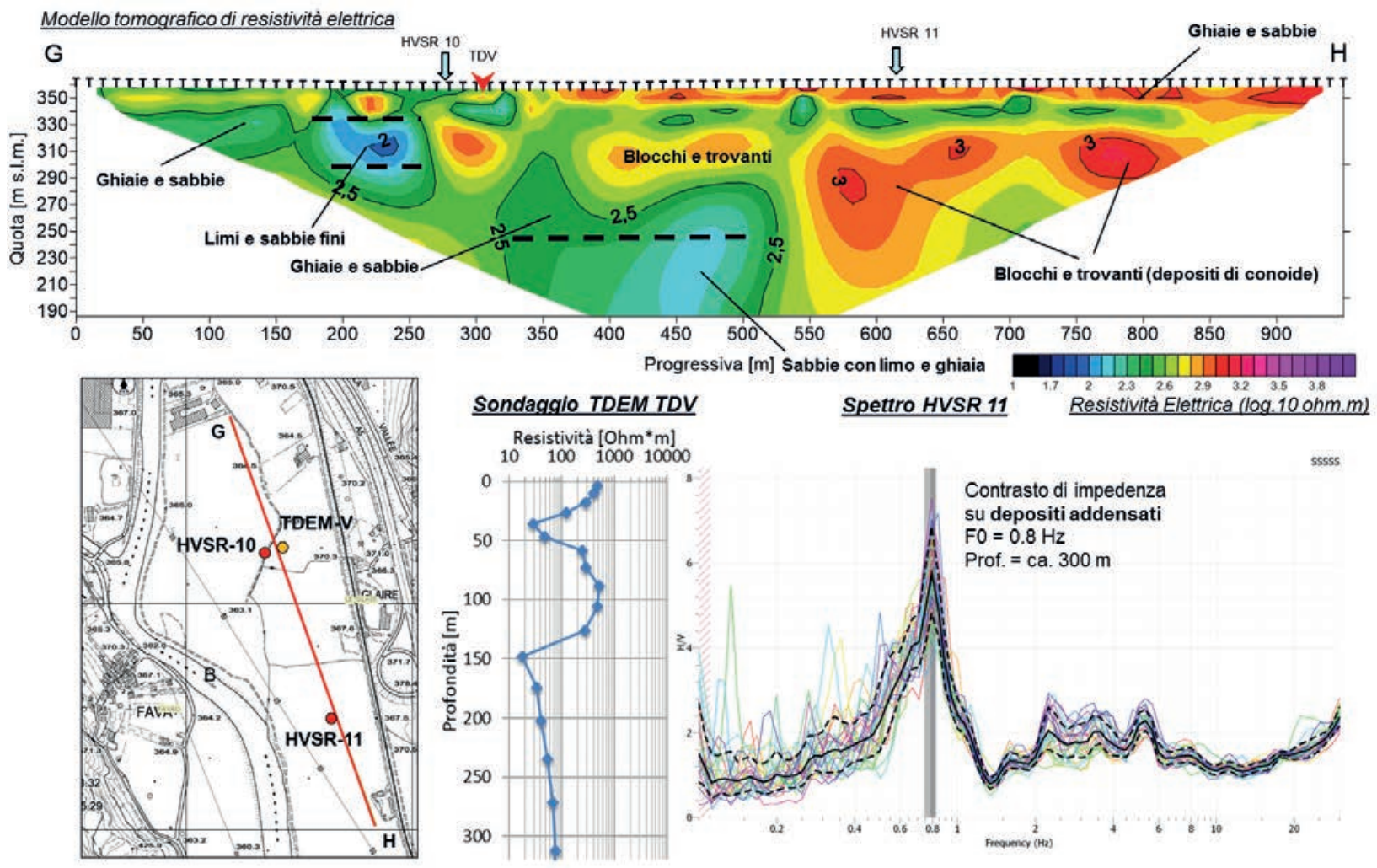

Fig. 9 - Investigations in the north Verrès site.

Fig. 9 - Indagini sul sito di Verrès nord

Nella porzione di valle dello stendimento si rilevano, al di sotto dei depositi alluvionali recenti, materiali ad alta resistività, interpretabili come depositi coalescenti di conoide ad elevata energia (blocchi lapidei), coalescenti del torrente Evançon, che rappresentano contributi laterali ai depositi fluvio-glaciali di fondovalle.
Dal sondaggio TDEM (riportato in dettaglio in Fig. 10) si evince uno spessore dei depositi alluvionali e glaciali superiore ai $300 \mathrm{~m}$; in particolare dai 150 m di profondità si rileva la presenza di materiale a minore resistività, a matrice più fine. Il sondaggio HVSR individua una frequenza di risonanza pari a $0.8 \mathrm{~Hz}$, cui corrisponde una profondità di circa $300 \mathrm{~m}$.

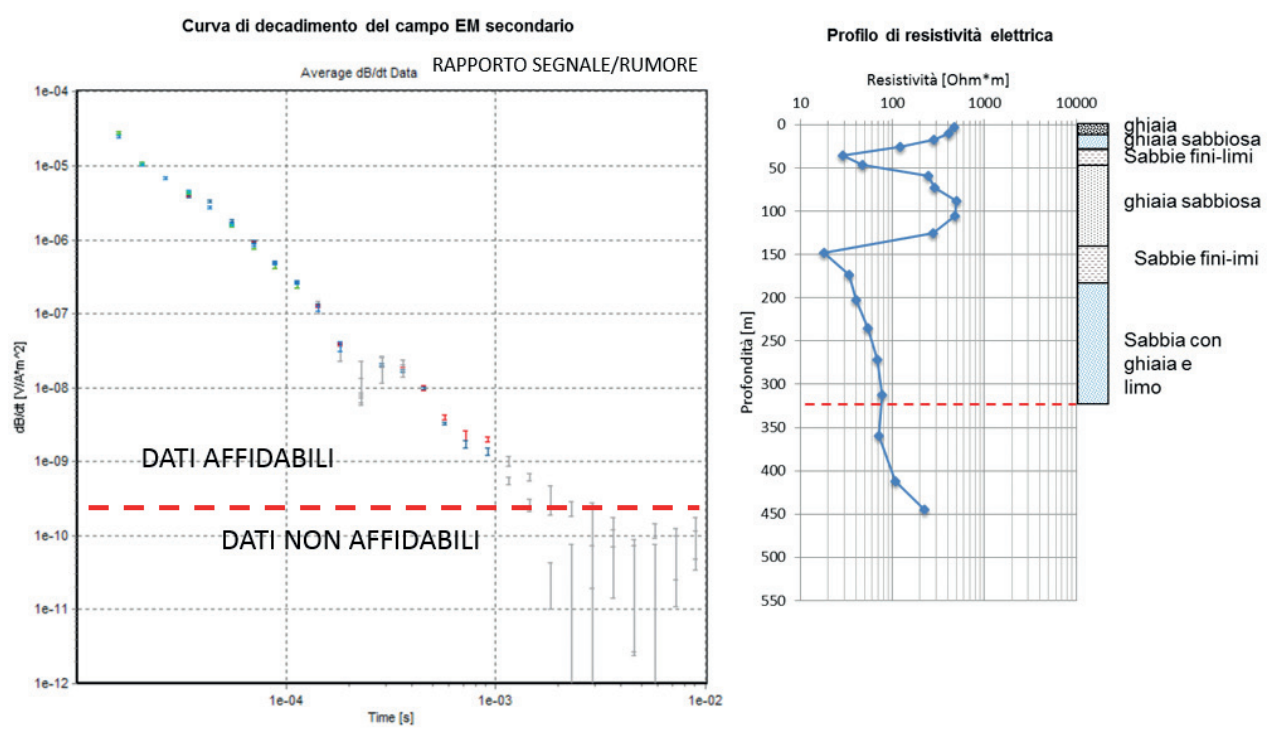

Fig. 10 - Detail of the TDEM north Verrès investigation and its interpretation.

Fig. 10 - Dettaglio dell'indagine TDEM Verrès nord e sua interpretazione. 


\section{Sito di Verrès Sud}

Sono stati eseguite le seguenti indagini (Fig. 11):

- $\quad$ n. 3 prove sismiche HVSR con tempo di registrazione di 4 ore;

- n. 1 stendimento geoelettrico lungo $950 \mathrm{~m}$.

La sezione ETR evidenzia, a partire dai 150 m di profondità, la presenza di depositi lacustri interdigitati con depositi molto grossolani (afflussi laterali), corrispondenti - in analogia con quanto rilevato a Verrès Nord - a depositi di conoide ad elevata energia (blocchi lapidei) provenienti da affluenti laterali.

Dalla prova HVSR si ricava uno spessore dei depositi alluvionali e fluvio-glaciali pari a circa $450 \mathrm{~m}$.

\section{Sito di Issogne}

Sono state eseguite le seguenti indagini (Fig. 12):

- n. 1 prova sismica HVSR;

- n. 1 stendimento geoelettrico lungo $950 \mathrm{~m}$.

In questo stendimento si assiste, rispetto ai due siti precedenti ubicati più a monte, ad un aumento dei depositi lacustri con riduzione degli apporti torrentizi laterali.

Anche in questo caso, la prova HVSR ha individuato il substrato lapideo ad una profondità di circa $400-450 \mathrm{~m}$ da piano campagna.

\section{Sito di Hône}

Sono state eseguite le seguenti indagini (Fig. 13):

- n. 10 sondaggi elettromagnetici TDEM (i cui dati sono fortemente disturbati e quindi inutilizzabili);
- $\quad$ n. 2 prove sismiche HVSR;

- n. 1 stendimento geoelettrico lungo $950 \mathrm{~m}$ :

- n. 1 prova Refraction Microtremor, finalizzata alla misura della velocità delle onde superficiali i cui risultati sono stati utilizzati per l'interpretazione di tutte le prove HVSR.

La sezione ERT evidenzia la presenza, a poche decine di m di profondità, di depositi fluvio-lacustri (limi e argille) frammisti a depositi grossolani (blocchi lapidei), corrispondenti a probabili interdigitazioni con scaricatori glaciali.

La prova HVSR conferma anche in questo caso il substrato a circa $400 \mathrm{~m}$. La prova TDEM (TH04) conferma la presenza dei livelli a grossi blocchi (fortemente resistivi) e fino a circa $350 \mathrm{~m}$ si rilevano valori di resistività elettrica congruenti con depositi fluvioglaciali/glaciali (100-200 ohm.m).

\section{Conclusioni}

Le indagini geofisiche eseguite nella piana di Verrès hanno evidenziato una sequenza stratigrafica complessa, caratterizzata da depositi alluvionali recenti (di potenza compresa tra 40 e 50 m e resistività elettrica superiore ai 1000 $\mathrm{ohm} \cdot \mathrm{m}$ ) che si sovrappongono a depositi lacustri (sabbie fini e limi a resistività elettrica compresa tra 10 e $100 \mathrm{ohm} \cdot \mathrm{m})$, a loro volta sovrastanti sedimenti fluvioglaciali e glaciali (ghiaie e sabbie con limo) (Fig. 14).

I depositi lacustri non risultano essere continui lateralmente, essendo interdigitali con $\mathrm{i}$ depositi fluvioglaciali, da mediamente a molto grossolani (apporti torrentizi laterali).

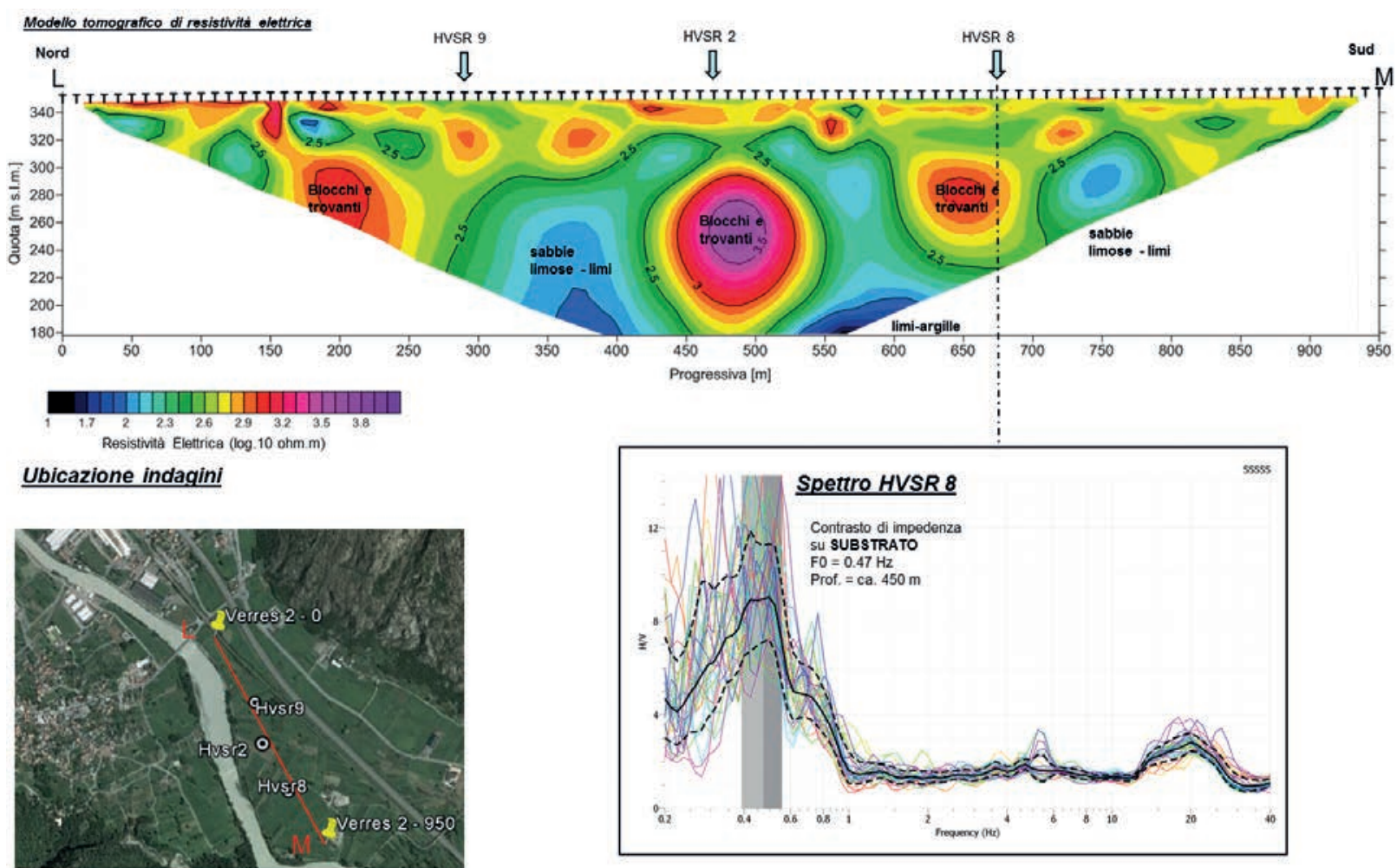

Fig. 11 - Investigations in the south Verrès site.

Fig. 11 - Indagini sul sito di Verrès sud. 


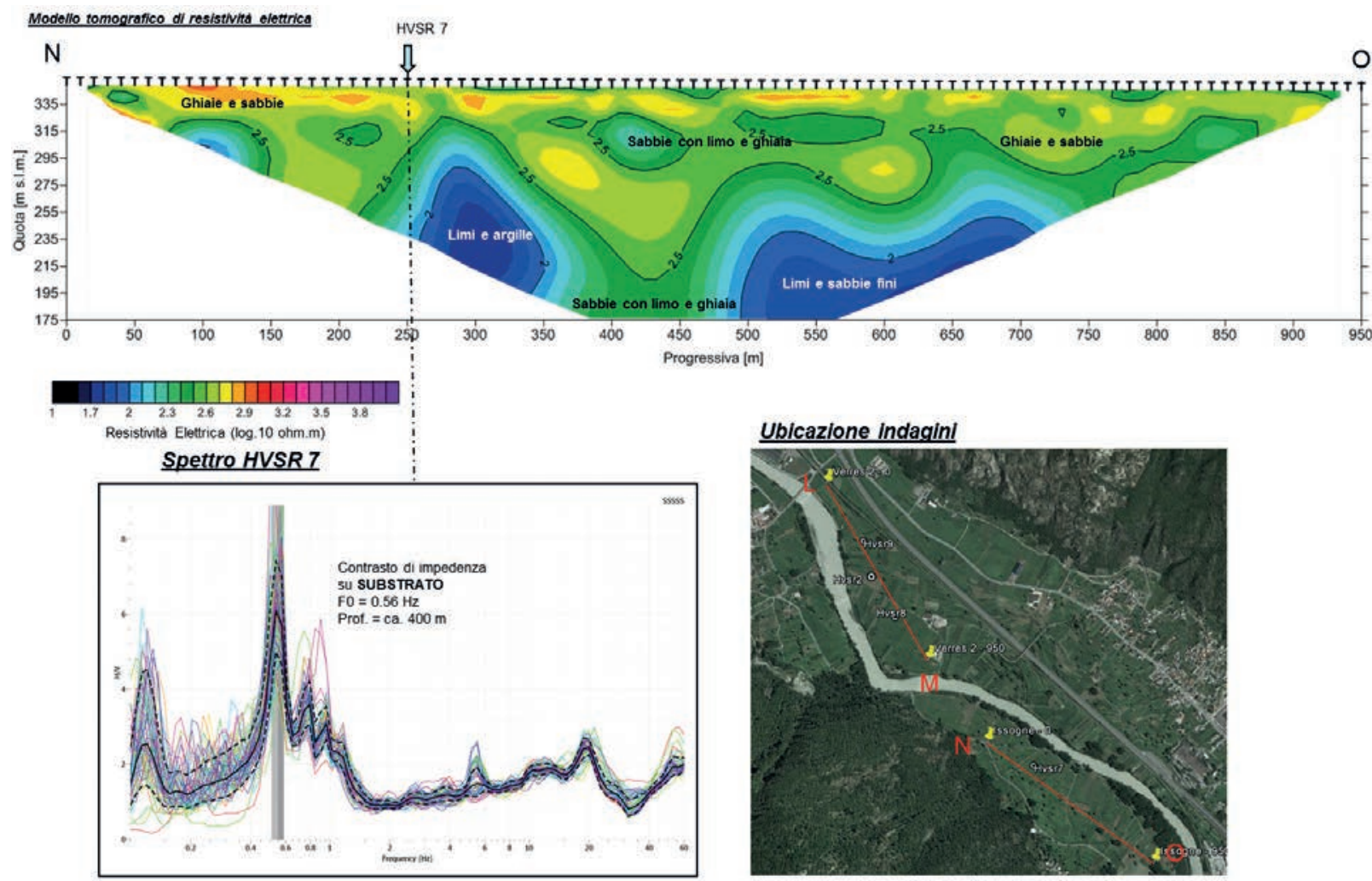

Fig. 12 - Investigations in the Issogne site.

Fig. 12 - Indagini sul sito di Issogne.

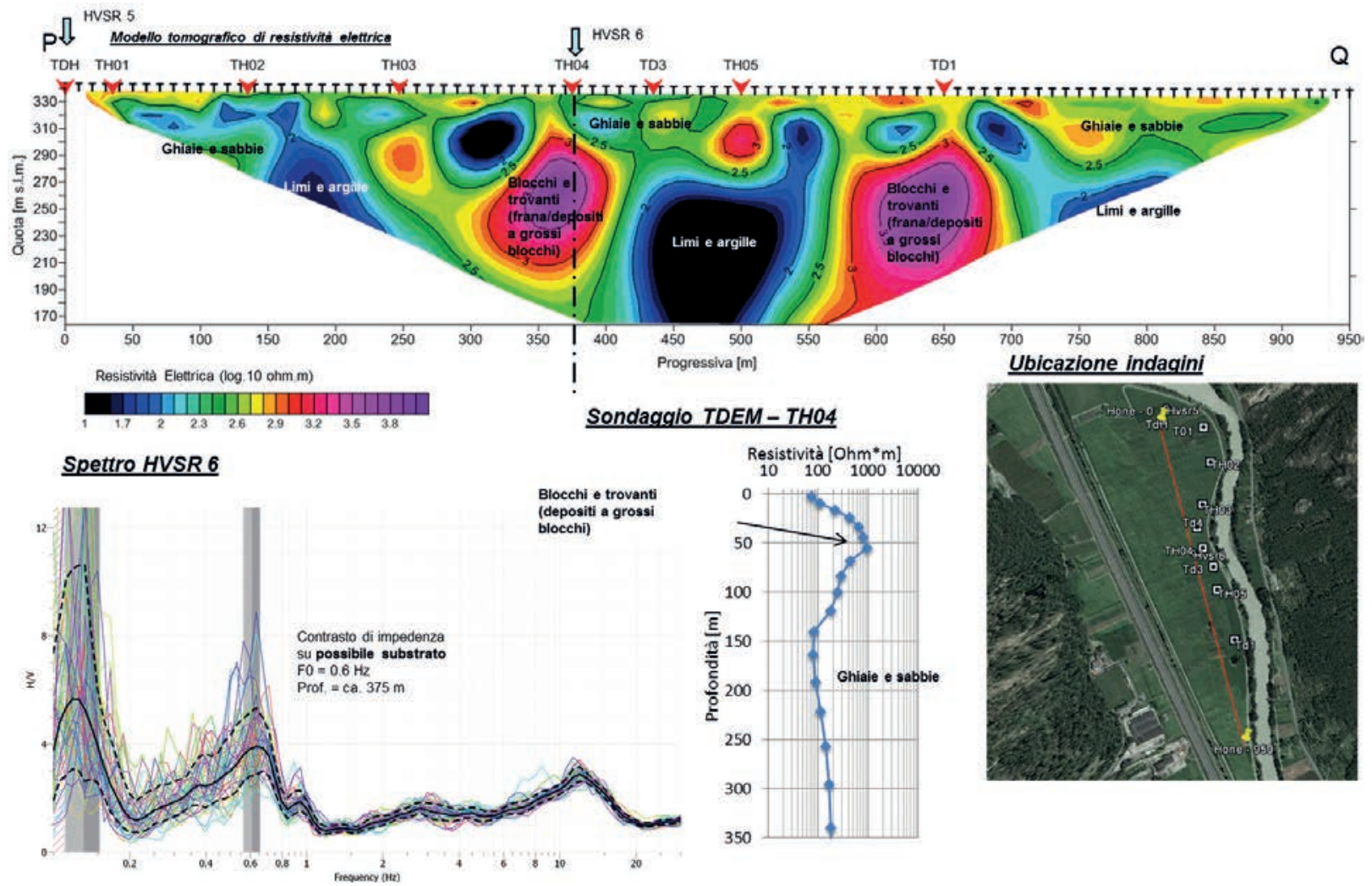

Fig. 13 - Investigations in the Hône site.

Fig. 13 - Indagini sul sito di Hône. 


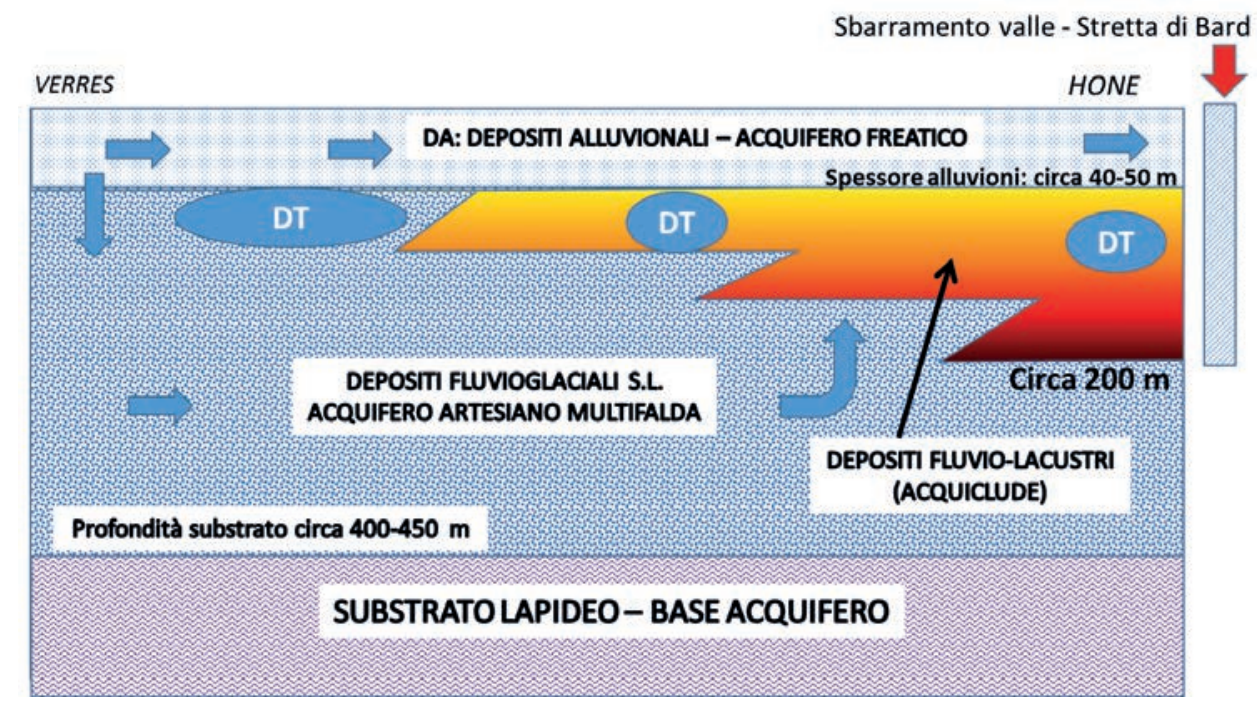

Fig. 14 - Simplified hydrogeological model -longitudinal section. DT = creek deposits. Arrows show main groundwater directions.

Fig. 14 - Modello idrogeologico semplificato - sezione longitudinale. DT = depositi torrentizi. Le frecce indicano le principali direzioni di deflusso delle acque sotterranee.

Tali dati confermano nella sostanza i risultati derivanti da analoghi studi geologici e geofisici effettuati su altri settori del fondovalle valdostano, in particolare nella piana di Aosta ove è stata riconosciuta l'esistenza di almeno due laghi che hanno occupato il fondovalle in epoca post-glaciale. (Fig.15)

Per la piana di Verrès pertanto sussistono ottime condizioni geologiche di reperimento idrico a profondità di indicativamente $200 \mathrm{~m}$ dal piano campagna in corrispondenza di depositi di origine glaciale. Tale presunto acquifero profondo avrebbe, appena a valle di Verrès, carattere artesiano in quanto delimitato e protetto a tetto da un potente orizzonte limo-sabbioso di origine fluvio-lacustre.
L'importanza strategica di questo acquifero profondo è evidente. Premesso infatti che l'acquifero freatico utilizzato non mostra, sulla base del monitoraggio istituzionale svolto da ARPA, alcun segno di sovra sfruttamento, ciononostante gli scenari climatici evocati per i prossimi decenni, con estinzione delle masse glaciali in quota, impongono sin da ora una riflessione sulle possibilità di futuro reperimento idrico alternativo.

E' pertanto opportuno valutare l'esecuzione di ulteriori indagini geologiche, di tipo diretto (fori pilota profondi, indicativamente 50 e $200 \mathrm{~m}$ ), finalizzate ad una caratterizzazione dei terreni profondi sottostanti la piana di Verrès nonché della qualità delle acque sotterranee in essi ospitate.

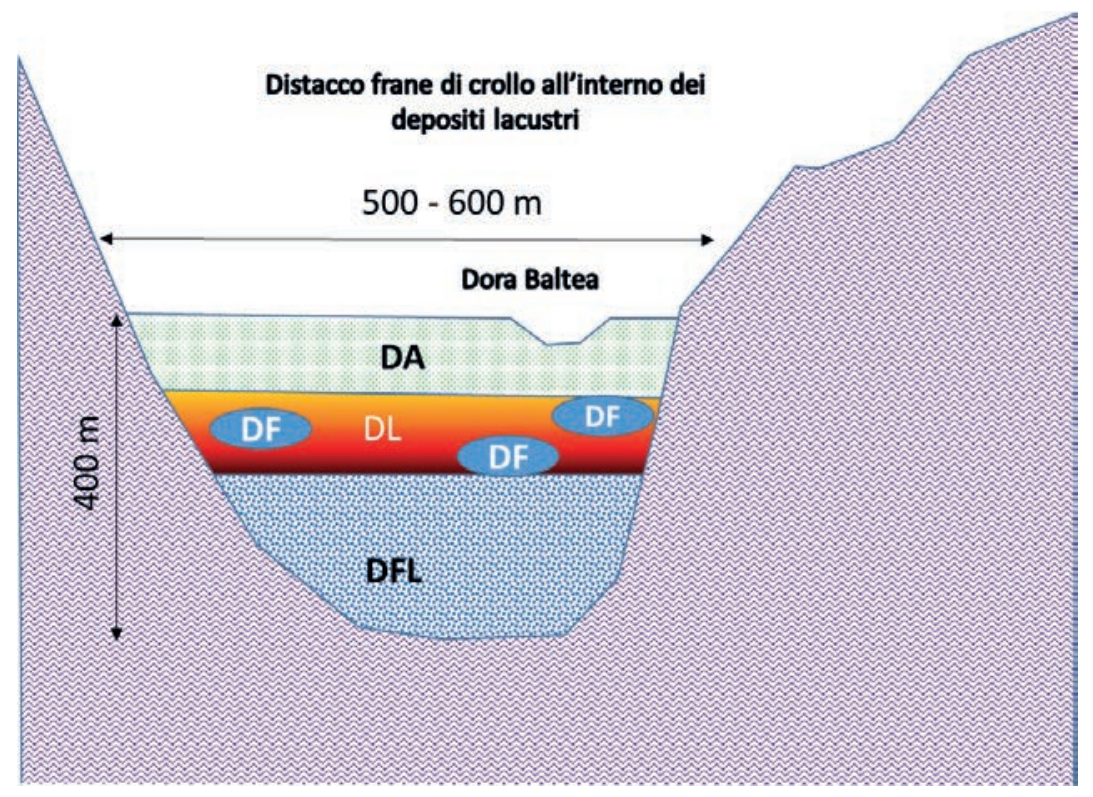

Fig. 15 - Simplified hydrogeological model - transversal section (Hône strait).

$D A=$ alluvial deposits (bigh recharge alluvial aquifer). $D L$ = lacustrine deposits. $D F L=$ fluvio-glacial deposits hosting a probable deep aquifer.

Fig. 15 - Modello idrogeologico semplificato - sezione traversale (stretta di Hône).

$\mathrm{DA}=$ depositi alluvionali (acquifero alluvionale a elevata ricarica). $\mathrm{DL}=$ depositi lacustri. $\mathrm{DFL}=$ depositi fluvio-glaciale ospitanti un probabile acquifero artesiano profondo. 


\section{BIBLIOGRAFIA}

Alfano L., Lorenzoni G., Melandri C., Mocchi C., Nuttini G., Pirera F., Soffientini M.E. (1994): A deep geoelectrical survey in the Southern Centrai Alps. Annali Di Geofisica, Vol. XXXVII, Suppl. N. 5, 1994

Armando E., Dal Piaz G.V. (1970) Studio geologico della coltre quaternaria nei dintorni di Aosta "Geological and Geophysical study of the quaternary layer near Aosta (preliminary observation)". Quaderno dell'Istituto di Ricerca sulle Acque, 1970: Ricerche sulle falde acquifere profonde delle valle alpine e appenniniche, 1-12

ARPA Valle d'Aosta (2014). Relazione tecnica sul monitoraggio annuale delle acque sotterranee,"Technical report about groundwater monitoring".

Benson, R. C. (2005). Remote sensing and geophysical methods for evaluation of subsurface conditions. Practical Handbook of Environmental Site Characterization and Ground-Water Monitoring, 249

Beres, Milan and Haeni, F.P. 1991. Applications of Ground Penetrating Radar Methods in Hydrogeologic Studies. Ground Water. v. 29, no. 3, pp. 375-386.

Bianquin N. (2010). Monitoraggio idrologico nella Regione Valle d'Aosta "Hydrological Monitoring in the Aosta Valley". Tesi di Laurea Magistrale in Geologia Applicata e Ambientale.Università degli Studi di Torino Facoltà di Scienze Matematiche, Fisiche e Naturali,

Borinatto M. (2014) Studio geologico e ambientale della piana di Verrès-Issogne-Arnad (AO) "Geological-environmental study of the VerrèsIssogne-Arnad Plain (Aosta Valley)". Tesi di laurea Magistrale in Geologia Applicata e Ambientale, Università degli Studi di Torino Facoltà di Scienze Matematiche, Fisiche e Naturali,

Brown C. J., Emily B. Voytek, John W. Lane, Jr., and Janet R. Stone Mapping Bedrock Surface Contours Using the Horizontal-toVertical Spectral Ratio (HVSR) Method Near the Middle Quarter Area, Woodbury, Connecticut - U.S. Department of the Interior U.S. Geological Survey - Open-File Report 2013-1028 Version 1.1, March 2013

Burger, H.R. (1992) Exploration Geophysics of the Shallow Subsurface. Prentice Hall PTR, Upper Saddle River, NJ.

Butler, D. K. (2005). Near-surface geophysics. Society of Exploration Geophysicists.
Elter P. (Agg. Bonetto-Dal Piaz- De Giusti) (2010) Carta geologica della Valle d'Aosta, scala 1:100.000 "Geological Map of the Aosta Valley". Tipografia Valdostana

ITALFERR \& PROMOGEO SRL (2011) - Indagini geognostiche e prove in sito - progetto preliminare ammodernamento ChivassoAosta tratta Verrès - Châtillon. Relazione tecnica "Geological investigations and in situ tests - preliminary poject of the Chivasso -Aosta railway, Verrès-Châtillon stertch. Technical report".

MELE Mauro, Martina BRUNO, Alfredo BINI, Davide TANTARDINI e Riccardo BERSEZIO La morfologia sepolta della confluenza tra Valchiavenna e Valtellina all'estremo Nord del Lago di Como, ricostruita per mezzo della prospezione sismica passiva (HVSR) "The buried morphology of the Valchiavenna-Valtellina confluence in the northern Como Lake, studied throughout passive seismic prospection (HVSR)". Geologia Insubrica. Volume 12 - n. 1 - 2016

Parasnis, D. S. (1997) Principles of Applied Geophysics ( ${ }^{\text {th }}$ edition), Chapman and Hall.

P.I.A.H.V.A.- Programme International d'Action Hydrogeologique en Val d'Aoste (1992). La nappe alluviale de la Doire - Vallée d'Aoste. "Alluvial groundwater of the Dora Baltea River (Aosta Valley)" Rapport finale premiere phase. Universites d'Avignon, Chambery, Turin, Centre de Recherches Geodynamiques de Tholon - Univ. Paris 6 - Ecole Polytecnique de Lausanne (GEOLEP).

Reynolds J.M.(1997) An Introduction to applied Environmental Geophysics, Wiley \& Sons, Baffins Lane.

TECHGEA (2013). Indagini geofisiche per l'esplorazione profonda del sottosuolo nella Piana di Verrès. Relazione tecnica. "Geophysical investigations aimed to the geological deep exploration in the Verrès Plain. Technical report"

U.S. Geological Survey (1986) Application of seismic-refraction techniques to hydrologic studies, Open-File Report, USGS Publications Warehouse, http://pubs.er.usgs.gov/publication/ofr84746

Zohdy A.A.R., Eaton G.P., and Mabey D.R. (1990) Techniques of Water Resources Investigations of the United States Geological Survey: Application of Surface Geophysics to Ground Water Investigations. Department of the Interior, United States Geological Survey. 